\title{
The Influence of Gravity on Single Crystallization in Large Volume Drops
}

\author{
Ion Sandu*, Iuliana Iordache, Claudiu Teodor Fleaca, Florian Dumitrache, \\ Ana Maria Niculescu \\ National Institute for Lasers, Plasma and Radiation Physics, Laser Department, Laser Photochemistry \\ Laboratory, Bucharest, Romania \\ Email: *ionsnd@yahoo.com, iuliana.iordache@inflpr, claudiu.fleaca@inflpr, florian.dumitrache@inflpr, \\ ana.niculescu@inflpr
}

Received 25 August 2014; revised 15 September 2014; accepted 6 October 2014

Copyright (C) 2014 by authors and Scientific Research Publishing Inc.

This work is licensed under the Creative Commons Attribution International License (CC BY).

http://creativecommons.org/licenses/by/4.0/

c) (i) Open Access

\begin{abstract}
The chemical, physical, and biological properties of more than two millions of proteins which follow to be synthesized by Pharmaceutical Industry, can be anticipated (by using their XRD diffractograms) if they will be grown from aqueous drops as high quality, large volume single-crystals. This is not a simple task and usually the growing process is seen as art rather than a science. The growing is expensive, time consuming, and finally an amorphous aggregate may result instead one single-crystal. In this article, we show for the first time how one single crystal can be grown in large volume hanging drops through their fast evaporation. The single nucleation is determined by choosing the proper sense of gravitational force relative to the drop triple line contact. In a special configuration, single-crystals of glycine and threonine were rapidly grown.
\end{abstract}

\section{Keywords}

Single Nucleation, Drop Evaporation, Protein Crystallization, Triple Line Contact, Interface, Gravity

\section{Introduction}

How complicated can a water drop be? Without interactions, it would be just a spherical reservoir of $\mathrm{H}_{2} \mathrm{O}$. However, when it interacts with a substrate, things start to change. For a partial wetting fluid on ideal substrates, its triple line contact (TLC) at equilibrium should have a well defined contact-angle $\theta$ determined by the Young's

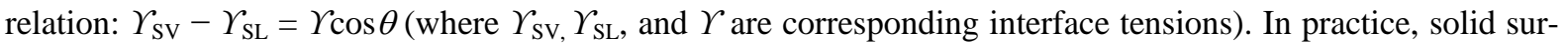
faces do not comply with Young's equation assumptions. Surfaces which are considered as atomically flat

"Corresponding author.

How to cite this paper: Sandu, I., Iordache, I., Fleaca, C.T., Dumitrache, F. and Niculescu, A.M. (2014) The Influence of Gravity on Single Crystallization in Large Volume Drops. Journal of Crystallization Process and Technology, 4, $206-211$.

http://dx.doi.org/10.4236/jcpt.2014.44025 
(silicon wafers, polymer films, etc.), induce some important phenomena such as: spreading [1] [2], pinning [2], contact angle hysteresis [2] which make the drop to take a variety of shapes: a sphere [3], a cape of sphere (Figure 1(a)) [3], a pancake like (Figure 1(b)) [3], or an infinity (mathematical) of other shapes if it is large, wets the substrate, and hangs on it (Figure 1(b)) [4]. In this article we will study only drops or droplets which partially wet the substrate $\left(\theta<90^{\circ}\right)$.

Now, let the drop to evaporate in still air. The problem becomes more complicated. If TLC is pinned on substrate, the drop keeps constant its radius $r$ and varies its $\theta$ (Figure 1(c)), and vice versa if elsewhere [5] [6]. A notable exception is the large volume hanging drops which evaporate, at least up to that they reach a cape of sphere shape, by keeping constant both $r$ and $\theta$ and varying their height $h$ [4]. On the other side, the rate of evaporation depends on relative humidity (RH) in the air [7] but also on the drop radius [8], or its surface or volume if it is a large one [9]. $\theta$ itself is not the same. It varies with the drop size [10] and RH [11]. When it reaches a critical value $\theta_{\mathrm{c}}$, the TLC deppinning [12] produces a sudden movement of TLC [12]. Here, we will study the evaporation in still air of pinned drops.

Let's complicate even more our problem. The drop will not be simply water but a nano or micro particle suspension. Now, a ring of particles forms at TLC because of the radial internal flow generated by evaporation rate gradient between the drop's edge and its top (Figure 1(d)). Discovered by R. Deegan et al. [8] and named as "coffee ring" effect, it becomes subject of research for an impressing number of works. Recent articles have revealed the importance of drop position relative to the substrate (sessile or hanging) on the characteristics of nanoparticle ring, showing that gravity could influence the "coffee ring" effect [13] [14]. In the present work, we will study the influence of gravity on the ring, when the solution contains more complex objects than nanoparticles.

The next (and last) step is to replace the inorganic particles in suspension with an aqueous solution of ionic salts, which through evaporation will crystallize. Besides spreading, pinning, contact angle hysteresis, coffee ring, radial internal flow of the liquid, evaporation, and many others, we will have a first order transition phase namely the crystallization phenomenon. Otherwise, the crystallization by slow evaporation of a drop is an old, simple, and well known technique which usually deals with small volume (up to few $\mu \mathrm{l}$ ), sessile drops. However, the studies on this set-up have been focused only on kinetic and thermodynamic phenomena within the drop (homogeneous nucleation [15] [16]) or by taking into account the nature and roughness of the substrate (heterogeneous nucleation [15] [16]). The only few studies on large volume (tens and hundreds of $\mu$ l) sessile drops showed that complex or simple crystallizing substances such as $\mathrm{NaCl}$ or other ionic salts form a ring of single crystals at TLC. By evaporating aqueous $\mathrm{NaCl}$ drops deposited on hydrophilic glass slides, N. Shaidzadeh-Bonn et al. observed a "ring like crystalline deposit in the drop's border at the end of drying". They considered that the crystalline deposit is generated by the coffee-ring effect [17]. C. R. Navarro and Doehne [18] found a similar ring in which the number of well developed crystals was less numerous at low relative humidity. However, if for

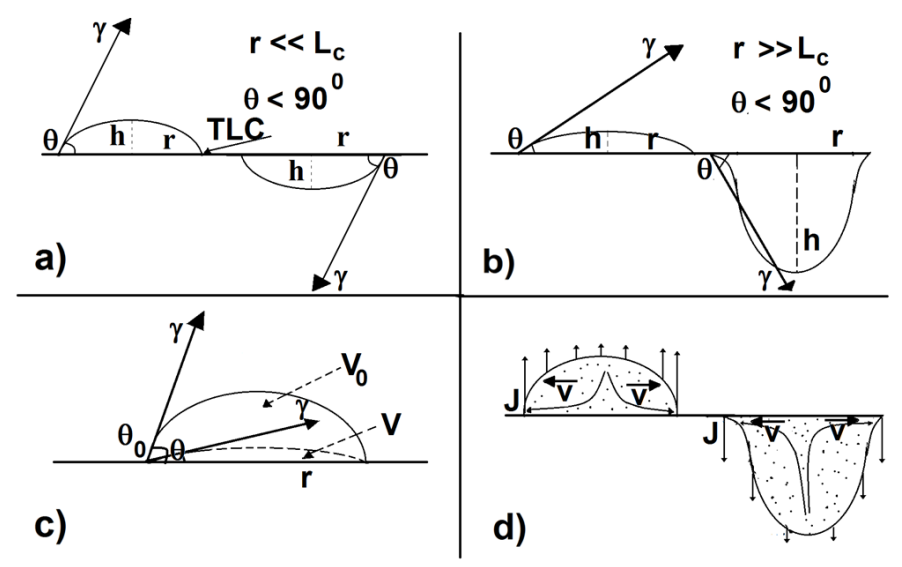

Figure 1. Shapes of aqueous drops on a hydrophilic substrate: (a) Cape of sphere; (b) Pancake (sessile), parabolic (hanging); (c) Constant contact radius evaporation of a pinned drop; (d) Coffee ring effect in a drop. 
large volume sessile drops we could access few articles, regarding the crystallization in large volume hanging drops we found no information. Concerning the influence of gravity, usually is considered as being too small comparing with all above mentioned phenomena [19] [20]. Crystallization experiments performed in reduced gravity [21] [22] or outer space [22] did not evidenced major differences in crystals structure or morphology. However, even if the gravity is normal or lower, it always acts from drop's top to its bottom. In a hanging drop, it acts in opposite direction and this, as we will see, is of a high importance for the nucleation phenomenon. We consider as important the study of such complex systems because, besides the better understanding of drop's interfaces phenomena, some time we need to grow only one, high quality, large single crystal within a drop (mononucleation or single crystallization [23]). This can happens when the crystallizing material is produced in a small quantity (protein) [24] and/or, it is thermally degradable (relative low processing temperature), and/or must be large enough $(>300 \mu \mathrm{m})$ for generating a high quality X-Ray diffractogram [24].

In this article, we show for the first time how large volume hanging drops can grow one single crystal per drop in the most common experimental conditions.

\section{Material and Methods}

Ionic salts and organic substances (Sigma, 99\% purity) were dissolved in distilled water near to their concentration of saturation, and their aqueous solutions were filtered through a $200 \mathrm{~nm}$ pore size filter. Drops with volumes varying between 10 - $300 \mu \mathrm{l}$ were deposited on a horizontal microscope slide glass and allowed to dry in still air ( $\mathrm{T} \sim 25^{\circ} \mathrm{C}$, RH was fixed between $20 \%$ and $70 \%$ ). Some drops were deposited in the interior of a special ring formed onto a microscope slide glass. It consists in radial spots of a hydrophobic substance (commercially available Poxypol ${ }^{\circledR}$ epoxy resin), sticked on the glass surface. The spot diameter is $\sim 1 \mathrm{~mm}$ and the distance between two opposite spots is $\sim 10 \mathrm{~mm}$. The pinned, large volume, hanging drops were deposited as it follows: a droplet $(5-10 \mu \mathrm{l})$ was deposited as sessile in the center of the polymeric ring. The substrate was turned upside down, now the droplet hanging on. With the tip of micro-syringe in contact with the drop, aqueous solution was slowly injected thus forming a large volume $(100-300 \mu \mathrm{l})$ drop. After the drop evaporation in still air, the as-grown single crystals were investigated through optical microscopy and X-Ray diffraction.

\section{Results and Discussion}

By evaporating a large number of aqueous $\mathrm{NaCl}$ drops, we observed that crystals in sessile drops nucleate at TLC and grow near it as one single crystal (in droplets) or as a crystalline ring (in large drops) (Figure 2), while in hanging drops the first single crystal which appears slip to the top of the drop forming one single crystal (Figure 3) or, remains stuck at TLC and forms an aggregate of crystals.

We found that this motion of the first growth crystal far away from TLC inhibits the growing of new crystals.

It is known that nucleation of first stable single crystal (few hundreds of micrometers in size) runs much more than its growing time [15] [16]. In order to nucleate a crystal, the solution must reach a specific critical supersaturation $S_{C}$ (defined as the ratio between the present concentration and solubility). This $S_{C}$, influences in an exponential manner the density of nucleation $J$ (the number of stable nuclei on unit of volume $V$, and time $t$ ) [15] [16] [25] (Figure 4(a)).

When the first (small) single crystal forms it grows rapidly, decreasing the supersaturation $S$ and thus decreasing the possibility that a new crystal to form in its vicinity [25]. However, this happens only if the single crystal

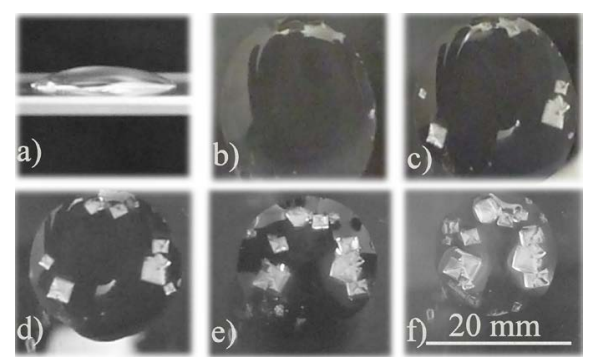

Figure 2. Single crystals ring formation on TLC: initial sessile drop; (a) Cross-section view; (b) Normal view; (c)-(f) Drop’s photo images during evaporation. 


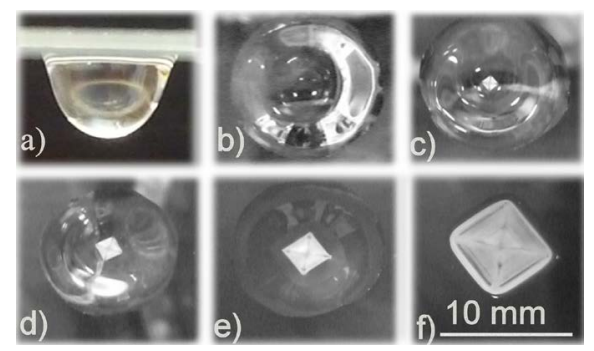

Figure 3. Single crystal formation on top of the drop: initial hanging drop; (a) Cross-section view; (b) Normal view; (c)-(f) Drop's photo images during evaporation.

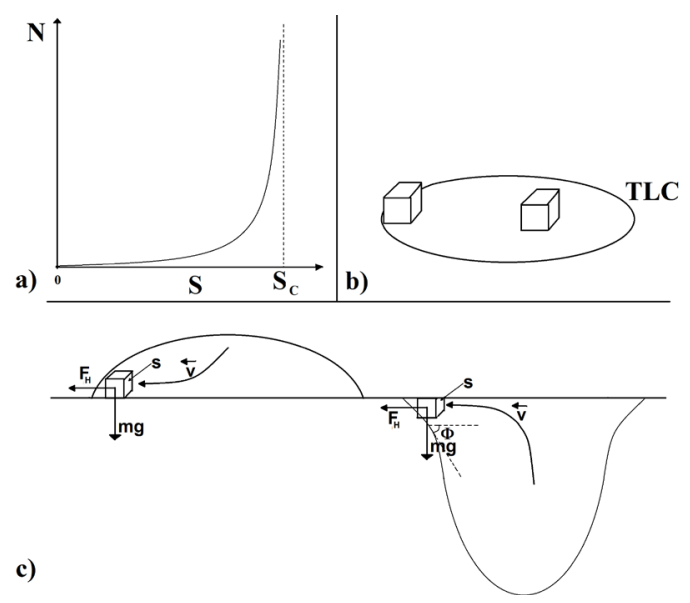

Figure 4. (a) The number of stable nuclei $N$, as a function of supersaturation $S$; (b) The first single crystal position in a drop; (c) The main forces which act on the crystal in a sessile and a hanging drop.

can be continuously "fuelled" with super-saturated solution. This depends on the diffusion rate of super-saturated solution from the bulk to the crystal and on crystal's position in the drop. A drop which grow at TLC will benefit by a lower quantity of supersaturated solution than one which grows somewhere in the bulk (Figure 4(b)). This means that in some zones, $S_{c}$ could be reached again, and another stable single crystal could form. This spatio-temporal concentration heterogeneity is produced by the evaporation rate gradient of the drop's surface and by the internal flow generated by this gradient [8]. A hanging drop, by its shape, offers the possibility of gravity-driven crystal movement faraway from TLC, thus by growing to decrease the $S$ in large surrounding zones, and consequently to decrease the probability that new single crystals to be generated in these zones (Figure 4(c)). In a large sessile drop this possibility is absent. The internal flow keeps the crystal near TLC, and the gravity acts in an inefficient way. The small drops, sessile or hanging, generate one single crystal per drop [23] because in this case, near TLC or on the top of the drop, the distance on which supersaturated solution fuels the crystal is comparable with the drop dimension (few hundreds of micrometers - the crystal, up to $1 \mathrm{~mm}$-the droplet) thus the concentration homogeneity is higher than in large crystals. Accepting the hypothesis that the position of the crystal inside the drop dictates the appearance of single crystallization, we could explain why the evaporation rate has so much influence. A high rate of evaporation will induce a higher internal radial flow of liquid, exerting an increased force on crystal, keeping it in the vicinity of TLC. A lower rate of evaporation will decrease this internal force and will made that the thermal motion to move the crystal (randomly) in a more efficient (regarding single crystallization phenomenon) zone.

If relatively isotropic (in dimension) crystals, fall down easily from TLC to the tip of a hanging drop, those which grow as long pins (needle-like), often have pinned their heads in TLC and in these cases the gravitation is insufficient for their pulling down to the drop's tip. Therefore, deposition of the hanging drop in the interior of hydrophobic ring (Figure 5(a), Figure 5(b)) leads to the increasing of $\theta$ and $\Psi$ (apparent angle) and thus the increasing of the drop surface slope will be more favourable to crystal slipping. Also, it may reduce the adhesion 


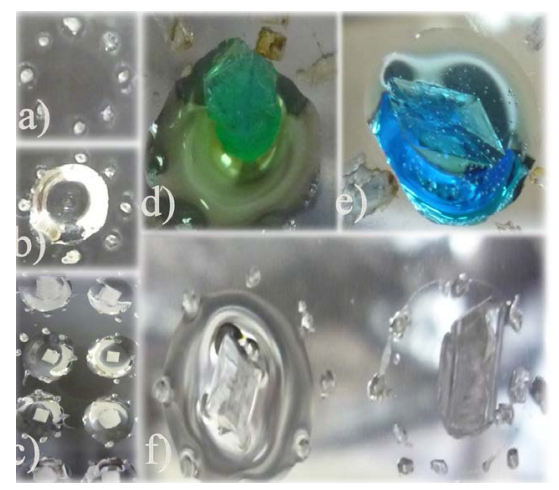

Figure 5. (a) Hydrophobic dots forming a ring; (b) An aqueous drop hanging in the interior of the ring; (c) Eight $\mathrm{NaCl}$ aq. hanging drops on the same substrate growing one single crystal within each drop; Large size single crystals grown from hanging drops using: (d) $\mathrm{CuSO}_{4}$; (e) $\mathrm{CuCl}_{2} \times$ $2 \mathrm{H}_{2} \mathrm{O}$; (f) Glycine $\left(\mathrm{NH}_{2} \mathrm{CH}_{2} \mathrm{COOH}\right)$.

strength which exists between crystal and substrate.

However, in this manner, the probability of single crystallization of aqueous $\mathrm{NaCl}$ increased up to $100 \%$ (Figure 5(c)), and substances which usually produce hundreds of small crystals could be grown as one large single crystal (Figures 5(d)-(f)). Unfortunately, not every substance that we tried crystallized as one single crystal. Some of them such as $\mathrm{FeSO}_{4}$, or citric acid remain as a highly viscous liquid. Fructose, glucose, and neomicin sulphate, crystallized as glassy solids. Other, reduced their number of grown single-crystals to a few, but never to a single one (at least in our experiments for $\mathrm{K}_{2} \mathrm{CrO}_{4}$, or ascorbic acid). This means that beside the crystal slipping, other phenomena influence the single crystallization. Even so, we consider that future studies of drops evaporation in experimental set-ups able to change well known paradigms, may lead to unexpected (and useful) results.

\section{Conclusion}

The so called "coffee ring" effect which appears during evaporation of colloidal sessile droplets, increases its complexity when a phase transition takes place in the drop. The drying of large volume aqueous drops of crystallizing substances has resulted in a ring of crystals if the drop is sessile; and one single-crystal if the drop hangs on the substrate. This difference is caused by gravity which in case of hanging drops can move the first crystallite which forms from triple line contact to drops' top. This new position of the growing crystal leads to the forming of one single crystal per each drop.

\section{References}

[1] Ramiasa, M., Ralston, J., Fetzer, R. and Sedev, R. (2014) The Influence of Topography on Dynamic Wetting. Advances in Colloid and Interface Science, 206, 275-293. http://dx.doi.org/10.1016/j.cis.2013.04.005

[2] Bonn, D. (2009) Wetting and Spreading. Reviews of Modern Physics, 81, 739-804. http://dx.doi.org/10.1103/RevModPhys.81.739

[3] Das, A.K. and Das, P.K. (2010) Equilibrium Shape and Contact Angle of Sessile Drops of Different Volumes-Computation by SPH and Its Further Improvement by DI. Chemical Engineering Science, 65, 4027-4037. http://dx.doi.org/10.1016/j.ces.2010.03.043

[4] Sumeh, P.T. and Guvindarajan, R. (2010) The Possible Equilibrium Shapes of Static Pendant Drops. The Journal of Chemical Physics, 133, Article ID: 144707. http://dx.doi.org/10.1063/1.3494041

[5] Song, H., Lee, Y., Jin, S., Kim, H.Y. and Yoo, J.Y. (2011) Prediction of Sessile Drop Evaporation Considering Surface Wettability. Microelectronic Engineering, 88, 3249-3255. http://dx.doi.org/10.1016/j.mee.2011.07.015

[6] Yu, Y.S., Wang, Z.Q. and Zhao, Y.P. (2013) Experimental Study of Evaporation of Sessile Water Droplet on PDMS Surfaces. Acta Mechanica Sinica, 29, 799-805. http://dx.doi.org/10.1007/s10409-013-0095-1

[7] Picknett, R.G. and Bexon, R. (1977) The Evaporation of Sessile or Pendant Drops in Still Air. Journal of Colloid and Interface Science, 61, 336-350. http://dx.doi.org/10.1016/0021-9797(77)90396-4 
[8] Deegan, R.D., Bakajin, O., Todd, F., Dupont, T.F., Huber, G., Nagel, R.S. and Witten, T.A. (1997) Capillary Flowasthe Cause of Ring Stains Fromdried Liquid Drops. Nature, 389, 827-829. http://dx.doi.org/10.1038/39827

[9] Murisic, N. and Kondic, L. (2011) On Evaporation of Sessile Drops with Moving Contact Lines. Journal of Fluid Mechanics, 679, 219-246. http://dx.doi.org/10.1017/jfm.2011.133

[10] Tadmor, R. and Yadav, P.S. (2008) As-Placed Contact Angles for Sessile Drops. Journal of Colloid and Interface Science, 317, 241-246. http://dx.doi.org/10.1016/j.jcis.2007.09.029

[11] Chhasatia, V.H., Joshi, A.S. and Sun, Y. (2010) Effect of Relative Humidity on Contact Angle and Particle Deposition Morphology of an Evaporating Colloidal Drop. Applied Physics Letters, 97, Article No. 231909. http://dx.doi.org/10.1063/1.3525167

[12] Ramos, S. and Tanguy, A. (2006) Pinning-Depinning of the Contact Line on Nanorough Surfaces. The European Physical Journal E, 19, 433-440. http://dx.doi.org/10.1140/epje/i2005-10056-0

[13] Sandu, I. and Fleaca, C.T. (2011) The Influence of Gravity on the Distribution of the Deposit Formed onto a Substrate by Sessile, Hanging, and Sandwiched Hanging Drop Evaporation. Journal of Colloid and Interface Science, 358, 621625.

[14] Hampton, M.A., Nguyen, T.A., Nguyen, A.V., Xu, Z.P., Huang, L. and Rudolph, V. (2012) Influence of Surface Orientation on the Organization of Nanoparticles in Drying Nanofluid Droplets. Journal of Colloid and Interface Science, 377, 456-462. http://dx.doi.org/10.1016/j.jcis.2012.03.024

[15] Sear, R.P. (2007) Nucleation: Theory and Applications to Protein Solutions and Colloidal Suspensions. Journal of Physics: Condensed Matter, 19, Article No. 033101. http://dx.doi.org/10.1088/0953-8984/19/3/033101

[16] Volmer, M. (1939) Kinetic der Phasenbildung (Steinkopff, Dresden, Leipzig).

[17] Shahidzadeh-Bonn, N., Rafai, S., Bonn, D. and Wegdam, G. (2008) Salt Crystallization during Evaporation: Impact of Interfacial Properties. Langmuir, 24, 8599-8605. http://dx.doi.org/10.1021/la8005629

[18] Rodriguez-Navarro, C. and Doehne, E. (1999) Salt Weathering: Influence of Evaporation Rate, Supersaturation and Crystallization Pattern. Earth Surface Processes and Landforms, 24, 191-209.

[19] Rusanov, A.I., Shchekin, A.K. and Tatyanenko, D.V. (2004) The Line Tension and the Generalized Young Equation: the Choice of Dividing Surface. Colloids and Surfaces A: Physicochemical and Engineering Aspects, 250, $263-268$. http://dx.doi.org/10.1016/j.colsurfa.2004.04.087

[20] Shapiro, B., Moon, H., Garell, R.L. and Kim, C.J. (2003) Equilibrium Behavior of Sessile Drops under Surface Tension, Applied External Fields, and Material Variations. Journal of Applied Physics, 93, 5794-5811. http://dx.doi.org/10.1063/1.1563828

[21] Erdmann, V.A., Lippmann, C., Betzel, C., Dauter, Z., Wilson, K., Hilgenfeld, R., et al. (1989) Crystallization of Proteins under Microgravity. FEBS Letters, 259, 194-198.

[22] Snell, E.H. and Helliwell, J.R. (2005) Macromolecular Crystallization in Microgravity. Reports on Progress in Physics, 68, 799-853. http://dx.doi.org/10.1088/0034-4885/68/4/R02

[23] Grossier R. and Veesler, S. (2009) Reaching One Single and Stable Critical Cluster through Finite-Sized Systems. Crystal Growth \& Design, 9, 1917-1922. http://dx.doi.org/10.1021/cg801165b

[24] Chayen, N.E. and Saridakis, E. (2008) Protein Crystallization: From Purified Protein to Diffraction-Quality Crystal. Nature Methods, 5, 147-153. http://dx.doi.org/10.1038/nmeth.f.203

[25] Garcia-Ruiz, J.M. (2003) Nucleation of Protein Crystals. Journal of Structural Biology, 142, 22-31. 
Scientific Research Publishing (SCIRP) is one of the largest Open Access journal publishers. It is currently publishing more than 200 open access, online, peer-reviewed journals covering a wide range of academic disciplines. SCIRP serves the worldwide academic communities and contributes to the progress and application of science with its publication.

Other selected journals from SCIRP are listed as below. Submit your manuscript to us via either submit@scirp.org or Online Submission Portal.
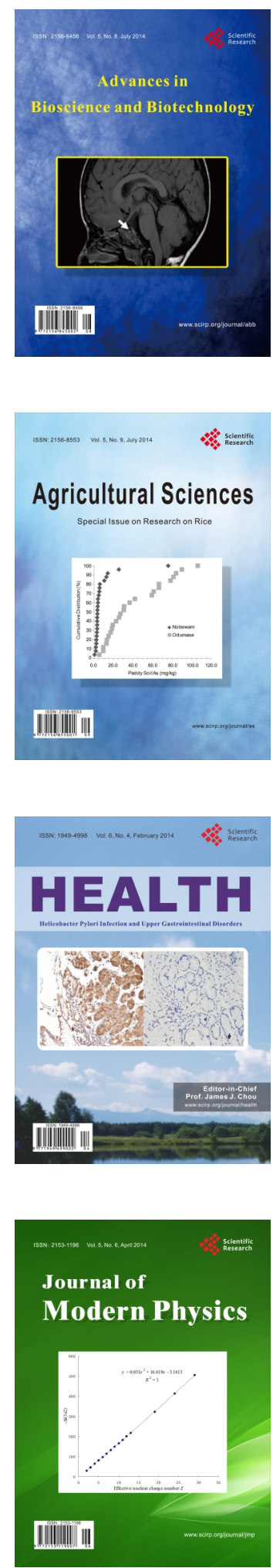
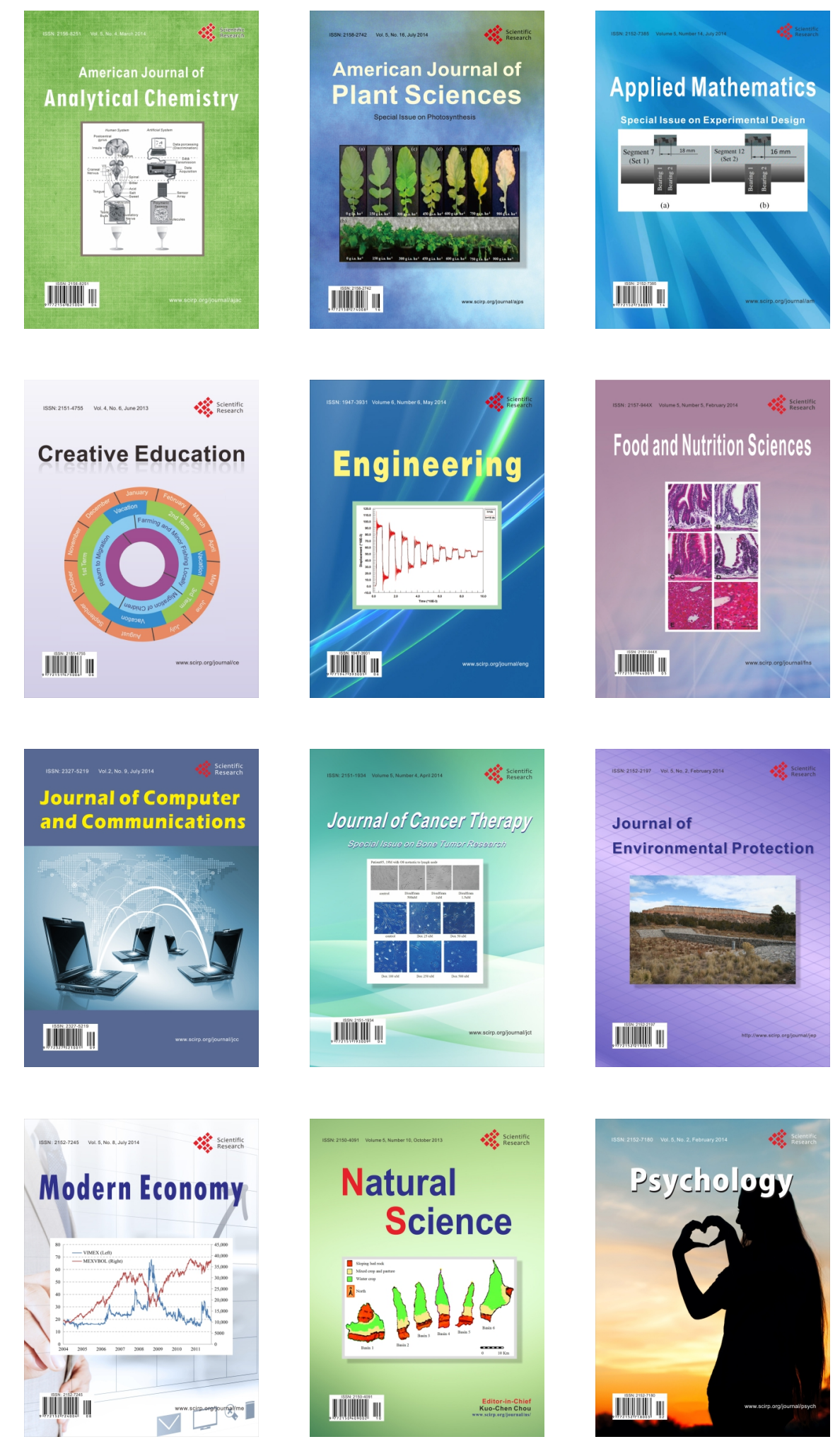